\title{
Microbiological Results From Middle Ear Effusion in Pediatric Patients Receiving Ventilation Tube Insertion: Multicenter Registry Study on the Effectiveness of Ventilation Tube Insertion in Pediatric Patients With Chronic Otitis Media With Effusion: Part I
}

\begin{abstract}
Myung Hoon Yoo ${ }^{1}$ Yang-Sun $\mathrm{Cho}^{2} \cdot$ June Choi ${ }^{3} \cdot$ Yun Hoon Choung ${ }^{4} \cdot$ Jae-Ho Chung ${ }^{5} \cdot$ Jong Woo Chung $^{6} \cdot$ Gyu Cheol Han $^{7}$

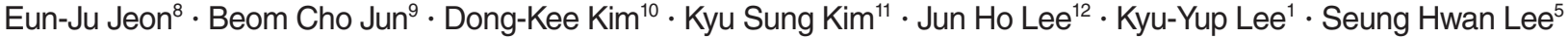
In Seok Moon ${ }^{13} \cdot$ Hong Ju Park ${ }^{6}$ Shi Nae Park ${ }^{14} \cdot$ Jihye Rhee ${ }^{3} \cdot$ Jae Hyun Seo $^{15} \cdot$ Seung Geun Yeo ${ }^{16}$

${ }^{1}$ Department of Otorhinolaryngology-Head and Neck Surgery, Kyungpook National University Hospital, School of Medicine, Kyungpook National University, Daegu; ' Department of Otorhinolaryngology-Head and Neck Surgery, Samsung Medical Center, Sungkyunkwan University School of Medicine, Seoul; ' ${ }^{3}$ Department of Otorhinolaryngology-Head and Neck Surgery, Korea University Ansan Hospital, Korea University College of Medicine, Ansan; ${ }^{4}$ Department of Otolaryngology, Ajou University School of Medicine, Suwon; ${ }^{5}$ Department of Otorhinolaryngology, Hanyang University School of Medicine, Seoul; ${ }^{6}$ Department of Otorhinolaryngology-Head and Neck Surgery, Asan Medical Center, University of Ulsan College of Medicine, Seoul; ' Department of Otolaryngology-Head and Neck Surgery, Gachon University of Medicine and Science, Graduate School of Medicine, Incheon; ${ }^{8}$ Department of Otolaryngology-Head and Neck Surgery, Incheon St. Mary's Hospital, College of Medicine, The

Catholic University of Korea, Incheon; ${ }^{9}$ Department of Otolaryngology-Head and Neck Surgery, Uijeongbu St. Mary's Hospital, College of Medicine, The Catholic University of Korea, Uijeongbu; ${ }^{10}$ Department of Otolaryngology-Head and Neck Surgery, Daejeon St. Mary's Hospital, College of Medicine, The Catholic University of Korea, Daejeon; ${ }^{11}$ Department of Otorhinolaryngology-Head and Neck Surgery, Inha University College of Medicine, Incheon; ${ }^{12}$ Department of Otorhinolaryngology, Seoul National University Hospital, Seoul National University College of Medicine, Seoul; ${ }^{13}$ Department of Otorhinolaryngology, Yonsei University College of Medicine, Seoul; ${ }^{14}$ Department of OtorhinolaryngologyHead and Neck Surgery, College of Medicine, The Catholic University of Korea, Seoul; ${ }^{15}$ Department of Otolaryngology-Head and Neck Surgery, Bucheon St. Mary's Hospital, College of Medicine, The Catholic University of Korea, Bucheon; ${ }^{16}$ Department of Otorhinolaryngology-Head and Neck Surgery, Kyung Hee University School of Medicine, Seoul, Korea
\end{abstract}

Objectives. The aim of this multicenter registry study was to investigate the effectiveness of ventilation tube insertion and the microbiology of otitis media with effusion (OME) in children. This part I study was conducted to evaluate the microbiological profile of children with OME who needed ventilation tube insertion.

Methods. Patients $<15$ years old who were diagnosed as having OME and received ventilation tube insertion were prospectively enrolled in 16 tertiary hospitals from June 2014 to December 2016. After excluding patients with missing data, the data of 397 patients were analyzed among a total of 433 enrolled patients. The clinical symptoms, findings of the tympanic membrane, hearing level, and microbiological findings were collected.

Results. In 103 patients (25.9\%), antibiotics were used within 3 weeks before surgery. Ventilation tube insertion was performed in a total of 710 ears (626 in both ears in 313 patients, 55 in the left ear only, and 29 in the right ear only). Culture of middle ear effusion was done in at least one ear in 221 patients $(55.7 \%)$, and in a total of 346 ears. Only 46 ears $(13.3 \%)$ showed positive results in middle ear effusion culture. Haemophilus influenzae $(17.3 \%$, followed by coagulase-negative Staphylococcus and Staphylococcus auricularis) was the most common bacteria detected.

Conclusion. $H$. influenzae was the most commonly found bacteria in middle ear effusion. Relatively low rates of culture positivity were noted in middle ear effusion of patients with OME in Korea.

Keywords. Otitis Media; Middle EarVentilation; Microbiology

- Received October 25, 2017, Revised January 19, 2018, Accepted January 25, 2018

- Corresponding author: Jong Woo Chung

Department of Otorhinolaryngology-Head and Neck Surgery, Asan Medical Center, University of Ulsan College of Medicine, 88 Olympic-ro 43-gil, Songpa-gu, Seoul 05505, Korea.Tel: +82-2-3010-3718, Fax: +82-2-489-2773, E-mail: jwchung@amc.seoul.kr

Copyright $\odot 2018$ by Korean Society of Otorhinolaryngology-Head and Neck Surgery.

This is an open-access article distributed under the terms of the Creative Commons Attribution Non-Commercial License (http://creativecommons.org/licenses/by-nc/4.0) which permits unrestricted non-commercial use, distribution, and reproduction in any medium, provided the original work is properly cited. 


\section{INTRODUCTION}

Otitis media is most common cause of acquired hearing impairment in children and affects two-thirds of all children within the first 3 years of life, with a cumulative incidence of $80 \%$ at the age of 4 years [1]. Prolonged hearing loss associated with otitis media with effusion (OME) may have a critical impact on language development [1-3]. As most cases of OME resolve spontaneously, watchful waiting, antibiotics, and surgical intervention (ventilation tube [VT] insertion) are the treatment options for OME $[4,5]$.

VT insertion is one of the most common operations performed in children and is considered the primary treatment of choice for OME when conservative management has failed for several months $[1,2,5]$.VT insertion can restore hearing immediately and can prevent frequent recurring flare-ups of otitis media. However,VT insertion requires general anesthesia (although for only a short duration) in young children and may have an increased risk for tympanic membrane problems including tympanosclerosis, persistent perforation, and atelectasis [1,2]. Therefore, selection of the appropriate treatment method in view of the risks and benefits of VT insertion is still controversial $[3,6,7]$.

Bacterial involvement in OME has been widely reported, with various available methods to identify pathogens from middle ear effusion, including traditional culture methods and polymerase chain reaction (PCR). Streptococcus pneumoniae, Haemophilus influenzae, and Moraxella catarrhalis are the most commonly isolated pathogens from OME; however, chronological changes in the bacteriology of OME have also recently been reported [8-10]. Therefore, the primary goal of this study was to evaluate the bacteriological profile of middle ear effusion in OME. In this study, we report the initial baseline data of the registry, especially focusing on the microbiology of the OME.

\section{MATERIALS AND METHODS}

\section{Study design}

This multicenter registry study (EVENT study, analysis of the effectiveness of VT insertion in pediatric patients with chronic otitis media: a multicenter registry study) has been ongoing since

\section{H I G H L I G H T S}

- Relatively low rate $(13.3 \%)$ of culture positivity were noted in middle ear effusion of patients with otitis media with effusion in Korea.

- Haemophilus influenzae was the most commonly found bacteria in middle ear effusion, followed by coagulase-negative Staphylococcus and Staphylococcus auricularis.

- The culture positive rate was not significantly different according to preoperative antibiotics use.
June 2014, using data from 16 tertiary hospitals throughout Korea. On the basis of previous studies [3,6,7], the target total number of patients was 500 . New patients were registered until December 2016, and follow-up data of registered patients will be collected until December 2017. The inclusion criteria were as follows: (1) age <15 years old and with OME that needs VT insertion; (2) diagnosis of OME based on the presence of middle ear effusion without signs of acute inflammation; (3) diagnosis was done with either an otoscope, pneumatic otoscope, microscope, endoscope, and/or tympanometer type B/C. Patients were excluded when they or their guardians refused enrollment. When VT was performed, culture of middle ear effusion was recommended. Data from the hospital records of patients who agreed to participate in the study were collected.

The study protocol was reviewed and approved by the Institutional Review Board of all hospitals. This protocol is registered in Clinical Research Information Service (http://cris.nih.go.kr, No. KCT0001378) for public access. Written informed consent was obtained from all parents/guardians and from children aged $>7$ years. All data were registered from each hospital by using an Internet-based electronic case report form (eCRF, http://otology.crf.kr).

\section{Measures}

As a part of the initial data, tympanic membrane findings, results of tympanometry, and results of pure tone audiometry were collected. Self-reported symptoms and systemic symptoms were also checked. History of surgery including previous VT insertion was reported. Moreover, information on recent antibiotics use (at 3 weeks before surgery, which is the timing when culture was performed) was also collected.

For data related to surgery, the status of middle ear effusion (serous, mucoid, glue, pus) and the type of VT were evaluated. If middle ear effusion culture was performed, the specific technique (needle aspiration, culture swab after myringotomy, or Juhn Tym-Tap aspiration) used to collect middle ear effusion was also identified. Culture reports and antibiotics susceptibility reports (only done for pathogenic bacteria) of the middle ear effusion were registered. In addition, complications related to surgery were reported. Follow-up data were also collected but not analyzed in this study.

\section{Statistics}

All statistical analyses were performed with SPSS ver. 18.0 (SPSS Inc., Chicago, IL, USA). Pearson chi-square test was used for all analysis. A $P$-value of $<0.05$ was considered to indicate statistical significance.

\section{RESULTS}

A total of 433 patients from 16 tertiary hospitals were regis- 
tered at the e-CRF site specifically for the EVENT study. Among them, 36 patients were excluded owing to missing data, and 397 patients (256 males, 141 females) were analyzed from the initial data. The mean patient age was $4.4 \pm 2.5$ years (range, 4 months to 14 years). Self-reported symptoms were present in 111 patients $(27.9 \%)$, mostly in older children. When the tympanic membrane was evaluated at the time of presentation, 40 patients (10.1\%) showed signs of acute inflammation. Pure tone audiometry was done in 146 patients (bone conduction in 101 of 146 patients) who were old enough to undergo the test. The mean bone conduction threshold was $7.5 \pm 7.7 \mathrm{~dB}$ in the right ear and $8.0 \pm 7.0 \mathrm{~dB}$ in the left ear. The mean air conduction threshold was $29.2 \pm 15.4 \mathrm{~dB}$ in the right ear and $27.9 \pm 12.7 \mathrm{~dB}$ in the left ear, showing an about $20 \mathrm{~dB}$ air-bone gap in both ears.

At the time of registration, VT was performed as a revision operation in 90 patients. VT was done in a total of 710 ears (both ears in 313 patients, left ear only in 55 patients, and right ear only in 29 patients). The status of effusion was serous in 233 patients (32.8\%), mucoid in $204(28.7 \%)$, glue in $207(29.2 \%)$, and purulent in $26(3.7 \%)$. A Paparella type I tube was inserted in 561 ears, Paparella type II tube in 14 ears, T-tube in 7 ears, and titanium collar-button-type tube in 126 ears. Overall, silicone-type VT was used in 582 ears $(82.0 \%)$ and titanium-type VT was used in 126 ears (18.0\%).

In 103 patients (25.9\%), antibiotics were used within 3 weeks before surgery. Intraoperative collection of middle ear effusion for culture was done in 221 patients ( $55.7 \%$; a total of 346 ears; both ears in 125 patients, left ear only in 44 patients, and right ear only in 52 patients). The specific technique used for collecting middle ear effusion for culture was needle aspiration in 120 ears $(34.9 \%)$, culture swab after myringotomy in 69 ears (20.1\%), or Juhn Tym-Tap aspiration in 155 ears (45.1\%).

Positive culture results were reported in 46 ears $(13.3 \%)$ of
36 patients. The detailed microbiology and antibiotic sensitivity results are shown in Table 1. One patient showed two pathogens present in one ear (Staphylococcus epidermidis and Candida parapsilosis). Moreover, there were two patients who had different microbiology findings between the two ears. When the culture positive rate was compared according to preoperative antibiotics use, ears from patients without use of preoperative antibiotics showed a positive rate of $12.6 \%$ and ears from patients who used preoperative antibiotics showed a positive rate of $16.5 \%$, and this difference was not statistically significant $(P>$ 0.05 ) (Fig. 1). The culture positive rate was also not significantly different among middle ear effusion types $(P>0.05)$ (Fig. 2). Furthermore, the culture positive rate was not statistically significant among the different specific techniques used to collect middle ear effusion (needle aspiration, $11.9 \%$; culture swab after myringotomy, 14.7\%; and Juhn Tym-Tap aspiration, $14.0 \%$; $P>$ 0.05 , Pearson chi-square test).

\section{DISCUSSION}

OME is known as a multifactorial disease, and Eustachian tube dysfunction, bacterial infection, allergy, and immunologic factors are considered its major causes [11,12]. Since Senturia and colleagues detected bacteria from middle ear effusion in 1958, bacterial infection has been known as an important factor in OME [13]. H. influenzae, S. pneumoniae, and M. catarrhalis were most common pathogens found in OME in several reports [8,13-15].

In our results, $H$. influenzae was also the most common (17.3\% of positive cultures) bacteria found in culture, followed by coagulase-negative Staphylococcus and Staphylococcus auricularis. S. pneumoniae and M. catarrhalis were both found in

Table 1. Detected bacteria in otitis media with effusion

\begin{tabular}{lccc}
\hline Bacteria & Patient & Ear & Antibiotics susceptibility \\
\hline Haemophilus influenzae & 6 & 8 & Multiple resistance in 1 patient (2 ears) \\
Coagulase-negative Staphylococcus & 6 & 8 & \\
Staphylococcus auricularis & 3 & 4 & Multiple resistance in 2 patients (2 ears) \\
Streptococcus mitis group & 3 & 4 & \\
Streptococcus pneumoniae & 3 & 3 & \\
Corynebacterium & 3 & 3 & Multiple resistance in 1 ear \\
Moraxella catarrhalis & 2 & 3 & Tetracycline resistance in 1 ear \\
Staphylococcus hominis & 2 & 3 & \\
Micrococcus group & 1 & 2 & \\
Streptococcus salivarius & 1 & 2 & \\
Streptococcus epidermidis and Candida parapsilosis & 1 & 1 & Multiple resistance (Streptococcus epidermidis) \\
Staphylococcus aureus & 1 & 1 & \\
Pseudomonas aeruginosa & 1 & 1 & \\
Acinetobacter Iwoffii & 1 & 1 & \\
Staphylococcus schleiferi & 1 & 1 & Multiple resistance in 1 ear \\
Aspergillus & 1 & 1 & \\
Total & 36 & 46 & \\
\hline
\end{tabular}




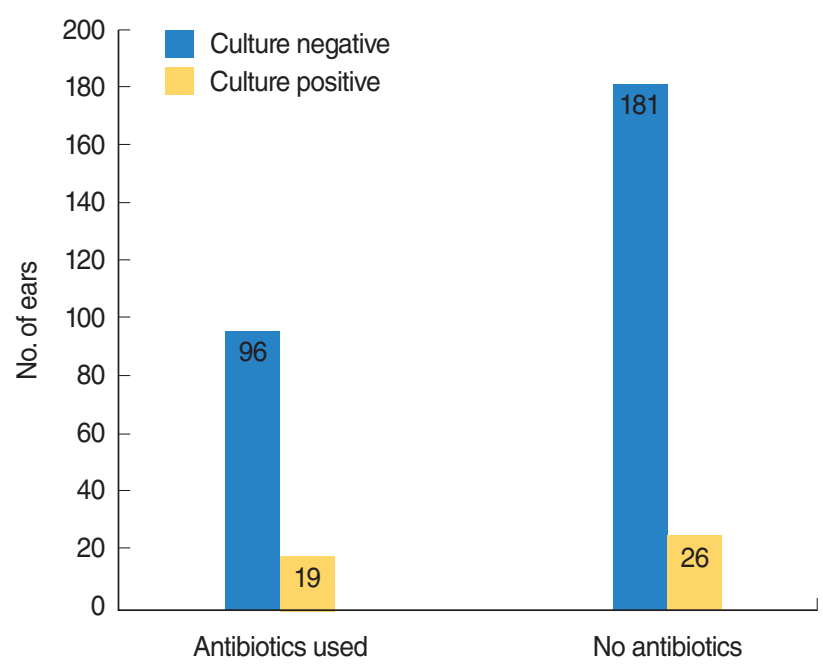

Fig. 1. Positive and negative culture results in each ear according to preoperative antibiotics use. There was no statistically significant difference of culture positive rate between two groups (antibiotics used vs. no antibiotics; $P>0.05$, Pearson chi-square test).

only $1 \%$ (each in three ears) of the total ears tested (each found in $6.5 \%$ among a total of 46 positive cultures). In recent studies about the changing patterns of bacterial strains in otitis media in Korea $[9,10]$, the detection rate of $S$. pneumoniae was found to have significantly decreased. Pneumococcal 7-valent conjugate vaccine (PCV7, Prevenar; Wyeth Pharmaceuticals, Philadelphia, PA, USA) was introduced in Korea in 2003 and implemented into national immunization programs for children $<5$ years old from 2014, and this may have reduced the identification of $S$. pneumoniae in otitis media in Korea [9]. However, as our survey did not investigate whether pneumococcal vaccinations were done previously in patients enrolled to the registry, it was not possible to analyze whether vaccination affected the positive culture rates. Since the introduction of PCV7, lower detection rates of $S$. pneumoniae and changes in the serotypes of this bacterium in OME have been reported $[9,16]$.

PCR detection methods are known to improve the detection rate of pathogens from 3.2-fold to 4.5-fold compared with the traditional culture method [16-20]; however, PCR data were not available in this study. Nevertheless, compared with previous reports in which traditional culture methods were used, our study still demonstrated relatively low rates of positive cultures from middle ear effusion. In a recent systemic review about the bacteriology of otitis media [16], the culture positive rate was $7 \%$ $36 \%$ in OME when the traditional culture method was used. Only results from Korea and Japan showed a range of $7 \%-10 \%$ positive detection rate, whereas other countries showed a rate of $19 \%-36 \%$. This may be due to various factors, including unidentified regional factors or the high rate of antibiotics use in OME. According to the "fourth report about propriety analysis for antibiotics use in pediatric acute otitis media" from the Health Insurance Review and Assessment Service of Korea, the

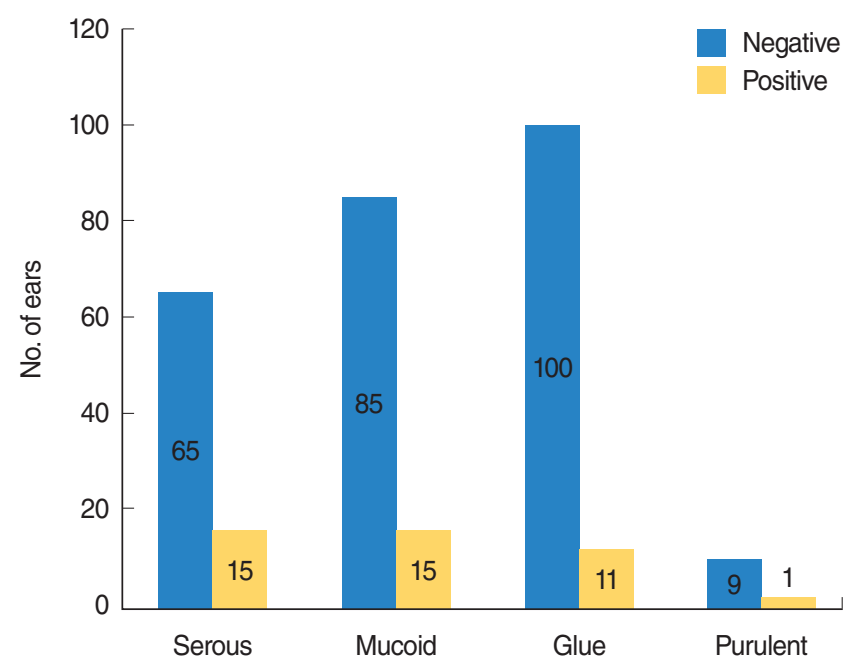

Fig. 2. Positive culture rates according to the status of middle ear effusion. There was no statistically significant difference of culture positive rate between the groups (serous vs. mucoid vs. glue vs. purulent; $P>0.05$, Pearson chi-square test).

rate of antibiotics prescription for acute otitis media in the pediatric population has decreased from $88.67 \%$ in 2012 to $84.19 \%$ in 2015. The antibiotics prescription rate in OME will be much lower than this; however, this rate in acute otitis media is still much higher than that reported in other countries ( $40 \%-50 \%$ in the Netherlands, $70 \%$ in Iceland, and $73 \%$ in Denmark) [21,22]. In about $25 \%$ of patients in our registry, antibiotics were used within 3 weeks before surgery, and this might have influenced the culture positive rates.

In our results, the microbiology of bilateral effusions among patients was mostly the same (in 10 of 12 patients who showed positive cultures from both ears). This agrees with previous reports that detected similar pathogens between two ears but frequently different pathogens from those found in adenoid tissue $[8,12,23]$. Although there were several nonpathogenic bacteria in our results and a positive culture does not always mean a definite pathogenic factor of OME, resistance to antibiotics was found in bacteria from eight ears $(17.4 \%$ of the total culture positive results) in six patients in our study, and those bacteria were resistant to multiple antibiotics in most cases. In these patients in whom resistant pathogens were identified, this information may guide future antibiotics use in case of post-tube otorrhea or recurrent acute otitis media.

The main limitation of our study is that it relied only on culture data without more specific techniques such as PCR or virus culture. Further results of the registry follow-up data will be reported at the end of the registry period after at least 1 year of follow-up in all patients. In summary, we report the initial baseline data of an ongoing multicenter registry study of OME in 433 patients. Our results showed a relatively low rate of positive cultures from middle ear effusion compared with other countries. H. influenzae followed by coagulase-negative Staphylo- 
coccus and S. auricularis were the most common pathogens detected in culture. To identify new pathogens involved in OME and to detect the development of antibiotic resistance, continuous monitoring of the bacteriology of otitis media is important.

\section{CONFLICT OF INTEREST}

No potential conflict of interest relevant to this article was reported.

\section{ACKNOWLEDGMENTS}

This work was supported by the Otitis Media Research Project funded by the Korean Otologic Society, Seoul, Republic of Korea.

\section{REFERENCES}

1. Lous J, Burton MJ, Felding JU, Ovesen T, Rovers MM, Williamson I. Grommets (ventilation tubes) for hearing loss associated with otitis media with effusion in children. Cochrane Database Syst Rev. 2005 Jan;(1):CD001801.

2. Hong HR, Kim TS, Chung JW. Long-term follow-up of otitis media with effusion in children: comparisons between a ventilation tube group and a non-ventilation tube group. Int J Pediatr Otorhinolaryngol. 2014 Jun;78(6):938-43.

3. Stenstrom R, Pless IB, Bernard P. Hearing thresholds and tympanic membrane sequelae in children managed medically or surgically for otitis media with effusion. Arch Pediatr Adolesc Med. 2005 Dec; 159(12):1151-6.

4. Venekamp RP, Burton MJ, van Dongen TM, van der Heijden GJ, van Zon A, Schilder AG. Antibiotics for otitis media with effusion in children. Cochrane Database Syst Rev. 2016 Jun;(6):CD009163.

5. Rosenfeld RM, Shin JJ, Schwartz SR, Coggins R, Gagnon L, Hackell JM, et al. Clinical practice guideline: otitis media with effusion (update). Otolaryngol Head Neck Surg. 2016 Feb;154(1 Suppl):S1-41.

6. Maw R, Wilks J, Harvey I, Peters TJ, Golding J. Early surgery compared with watchful waiting for glue ear and effect on language development in preschool children: a randomised trial. Lancet. 1999 Mar;353(9157):960-3.

7. Paradise JL, Feldman HM, Campbell TF, Dollaghan CA, Colborn DK, Bernard BS, et al. Effect of early or delayed insertion of tympanostomy tubes for persistent otitis media on developmental outcomes at the age of three years. N Engl J Med. 2001 Apr;344(16): 1179-87.

8. Chan CL, Wabnitz D, Bardy JJ, Bassiouni A, Wormald PJ, Vreugde S, et al. The microbiome of otitis media with effusion. Laryngoscope. 2016 Dec;126(12):2844-51.

9. Kim H, Choo OS, Jang JH, Park HY, Choung YH. Chronological changes in microbial profiles in external and middle ear diseases: a 20-year study in Korea. Eur Arch Otorhinolaryngol. 2017 Mar;274 (3):1375-81.

10. Lee JS, Kim MG, Hong SM, Na SY, Byun JY, Park MS, et al. Changing patterns of bacterial strains in adults and children with otitis media in Korean tertiary care centers. Clin Exp Otorhinolaryngol. 2014 Jun;7(2):79-86.

11. Almac A, Elicora SS, Yumuk Z, Dundar V, Willke A. The relationship between chronic otitis media with effusion and surface and deep flora of hypertrophic adenoids. Int J Pediatr Otorhinolaryngol. 2009 Oct;73(10):1438-40.

12. Eser OK, Ipci K, Alp S, Akyol U, Unal OF, Hascelik G, et al. Efficacy of nasopharyngeal culture in identification of pathogen in middle ear fluid in chronic otitis media with effusion. Indian J Med Microbiol. 2009 Jul-Sep;27(3):237-41.

13. Park CW, Han JH, Jeong JH, Cho SH, Kang MJ, Tae K, et al. Detection rates of bacteria in chronic otitis media with effusion in children. J Korean Med Sci. 2004 Oct;19(5):735-8.

14. Poetker DM, Lindstrom DR, Edmiston CE, Krepel CJ, LinkTR, Kerschner JE. Microbiology of middle ear effusions from 292 patients undergoing tympanostomy tube placement for middle ear disease. Int J Pediatr Otorhinolaryngol. 2005 Jun;69(6):799-804.

15. Jung H, Lee SK, Cha SH, Byun JY, Park MS, Yeo SG. Current bacteriology of chronic otitis media with effusion: high rate of nosocomial infection and decreased antibiotic sensitivity. J Infect. 2009 Nov; 59(5):308-16.

16. Ngo CC, Massa HM, Thornton RB, Cripps AW. Predominant bacteria detected from the middle ear fluid of children experiencing otitis media: a systematic review. PLoS One. 2016 Mar;11(3):e0150949.

17. Brook I, Yocum P, Shah K, Feldman B, Epstein S. Microbiology of serous otitis media in children: correlation with age and length of effusion. Ann Otol Rhinol Laryngol. 2001 Jan;110(1):87-90.

18. Shishegar M, Faramarzi A, KazemiT, Bayat A, Motamedifar M. Polymerase chain reaction, bacteriologic detection and antibiogram of bacteria isolated from otitis media with effusion in children, Shiraz, Iran. Iran J Med Sci. 2011 Dec;36(4):273-80.

19. Jero J, Virolainen A, Salo P, Leinonen M, Eskola J, Karma P. PCR assay for detecting Streptococcus pneumoniae in the middle ear of children with otitis media with effusion. Acta Otolaryngol. 1996 Mar;116(2):288-92.

20. Matar GM, Sidani N, Fayad M, Hadi U. Two-step PCR-based assay for identification of bacterial etiology of otitis media with effusion in infected Lebanese children. J Clin Microbiol. 1998 May;36(5): 1185-8.

21. Run Sigurdardottir N, Nielsen AB, Munck A, Bjerrum L. Appropriateness of antibiotic prescribing for upper respiratory tract infections in general practice: comparison between Denmark and Iceland. Scand J Prim Health Care. 2015 Dec;33(4):269-74.

22. Uijen JH, Bindels PJ, Schellevis FG, van derWouden JC. ENT problems in Dutch children: trends in incidence rates, antibiotic prescribing and referrals 2002-2008. Scand J Prim Health Care. 2011 Jun; 29(2):75-9.

23. Buzatto GP, Tamashiro E, Proenca-Modena JL, Saturno TH, Prates MC, Gagliardi TB, et al. The pathogens profile in children with otitis media with effusion and adenoid hypertrophy. PLoS One. 2017 Feb; 12(2):e0171049. 